\begin{tabular}{|c|l|}
\hline Title & Knockdown of legumain inhibits cleavage of annexin A 2 in the mouse kidney \\
\hline Author(s) & $\begin{array}{l}\text { Yamane, Takuya; Hachisu, Rei; Y uguchi, Motoki; Takeuchi, Keisuke; Murao, Sato; Y amamoto, Y oshio; Ogita, } \\
\text { Hisakazu; Takasawa, Toshihide; Ohkubo, Iwao; A riga, Hiroy oshi }\end{array}$ \\
\hline Citation & $\begin{array}{l}\text { BIOCHEMICAL A ND BIOPHY SICA L RESEA RCH COMMUNICA TIONS, 430(2), 482-487 } \\
\text { https://doi.org/L0.1016j.bbrc.2012.12.010 }\end{array}$ \\
\hline Issue Date & 2013-01-11 \\
\hline Doc URL & http://hdl.handle.net/2115/52625 \\
\hline Type & article (author version) \\
\hline File Information & Manuscript with Title Page 1.pdf \\
\hline
\end{tabular}

Instructions for use 


\section{Knockdown of legumain inhibits cleavage of annexin A2 in the mouse kidney}

Takuya Yamane $^{\mathrm{a}^{*}}$, Rei Hachisu ${ }^{\mathrm{b}}$, Motoki Yuguchi ${ }^{\mathrm{b}}$, Keisuke Takeuchi $^{\mathrm{c}}$, Sato Murao ${ }^{\mathrm{a}}$, Yoshio Yamamoto $^{\mathrm{d}}$, Hisakazu Ogita ${ }^{\mathrm{c}}$, Toshihide Takasawa ${ }^{\mathrm{b}}$, Iwao Ohkubo ${ }^{\mathrm{e} *}$ and Hiroyoshi Ariga ${ }^{\mathrm{a}}$

${ }^{a}$ Graduate School of Pharmaceutical Sciences, Hokkaido University, Kita-ku, Sapporo 060-0812, Japan

${ }^{b}$ Division of Bioscience, Hokkaido System Science Co. Ltd., Kita-ku, Sapporo 001-0932, Japan

${ }^{c}$ Division of Molecular Medical Biochemistry, Department of Biochemistry and Molecular Biology, Shiga University of Medical Science, Seta, Otsu 520-2192, Japan

${ }^{d}$ Department of Ecology and Molecular Biology, Mie University, Iga, Mie 518-0131, Japan

${ }^{e}$ Department of Nutrition, School of Nursing and Nutrition, Tenshi College, Higashi-ku, Sapporo 065-0013, Japan

* Corresponding authors:

Takuya Yamane

Graduate School of Pharmaceutical Sciences, Hokkaido University, Sapporo 060-0812, Japan Tel: +81-11-706-3711; Fax: +81-11-706-4988; E-mail: t-yamane@pharm.hokudai.ac.jp

Iwao Ohkubo

Department of Nutrition, School of Nursing and Nutrition, Tenshi College, Higashi-ku, Sapporo 065-0013, Japan

Tel: +81-11-741-1051 (ext. 223); E-mail: ohkubo@tenshi.ac.jp 


\begin{abstract}
Legumain (EC 3.4.22.34) is an asparaginyl endopeptidase. Strong legumain activity was observed in the mouse kidney, and legumain was highly expressed in tumors. We previously reported that bovine kidney annexin A2 was co-purified with legumain and that legumain cleaved the N-terminal region of annexin A2 at an Asn residue in vitro. In this study, to determine whether annexin A2 is cleaved by legumain in vivo, siRNA-lipoplex targeting mouse legumain was injected into mouse tail veins. Mouse kidneys were then isolated and the effect of knockdown of legumain expression on annexin A2 cleavage was examined. The results showed that both legumain mRNA and protein expression levels were decreased in the siRNA-treated mouse kidneys and that legumain activity toward a synthetic substrate, Z-Ala-Ala-Asn-MCA, was decreased by about $40 \%$ in the kidney but not in the liver or spleen. Furthermore, cleavage of annexin A2 at the N-terminal region was decreased in the mouse kidney that had been treated with the legumain siRNA-lipoplex. These results suggest that legumain siRNA was delivered to the kidney by using LipoTrust and that the reduced legumain expression inhibited legumain-induced degradation of annexin A2 in vivo.
\end{abstract}

Key Words: Legumain, annexin A2, cationic liposome, siRNA, in vivo, mouse kidney 


\section{Introduction}

Legumain (EC 3.4.22.34) is an asparaginyl endopeptidase belonging to the cysteine peptidase C13 family [1]. Legumain activity has been detected in a number of mammalian tissues, including the kidney, placenta, spleen, liver and testis, and the highest level of activity was detected in the kidney [2]. We previously reported that legumain is mainly expressed in proximal tubules of the rat kidney [3]. We further reported that legumain might have an important role in remodeling of the extracellular matrix through degradation of fibronectin in renal proximal tubular cells [4]. It has recently been suggested that legumain plays an important role in tumor growth/metastasis, carotid artery-atherosclerosis development [5-10], hemophagocytic syndrome [11] and formation of human unstable carotid plaque [12].

Annexin A2, also named annexin II, calpactin 1, lipocortin II, chromobindin 8 and placental anticoagulant protein IV, was co-purified with legumain from the bovine kidney, and co-purified annexin A2 lacked the $\mathrm{N}$-terminal region [3]. The $\mathrm{N}$-terminal region of annexin A2 contains both p11 subunit-binding sites required for hetero-tetramer formation of annexin A2 and phosphorylation sites of $\mathrm{pp} 60^{\mathrm{src}}$ and protein kinase C (PKC) [13]. Annexin A2 is abundantly expressed in the receptor-recycling compartments of rat liver hepatocytes [14] and is required for aquaporin 2 (AQP2) trafficking in renal cells [15, 16]. Furthermore, it has been shown that the N-terminal region of annexin A2 has a role in endosomal membrane fusion [17] and that phosphorylation of Tyr-23 is essential for proper endosomal association and for annexin A2 function [18].

Cationic liposomes have been used in drug delivery systems, and LipoTrust, a novel cationic liposome, has been used as an efficient delivery system in vivo [19, 20]. In this study, to examine annexin A2 degradation by legumain in vivo, siRNA-lipoplex targeting mouse legumain was injected into the mouse tail veins. The results showed that both the expression levels of legumain mRNA and protein and the legumain activity toward a synthetic substrate 
were decreased and that degradation of annexin A2 at an N-terminal Asn residue was inhibited in legumain siRNA-lipoplex-treated mouse kidneys, indicating an important role of legumain in annexin A2 functions.

\section{Materials and Methods}

\subsection{Materials}

Sequences of siRNA targeting mouse legumain were designed by Hokkaido System Science (Sapporo, Japan) using the B-Algo ${ }^{\mathrm{TM}}$ algorithm. The sense and antisense strands of siRNAs and LipoTrust ${ }^{\mathrm{TM}} E X$ Oligo were also chemically synthesized by Hokkaido System Science. NIH3T3 cells were purchased from American Tissue Culture Collection (Manassas, VA, USA). RNAlater and RNeasy Mini were purchased from Qiagen (Venlo, Netherlands). SuperScript III, Bioanalyzer capillary chip and Z-Ala-Ala-Asn-MCA were obtained from Invitrogen (St. Louis, MO, USA), Agilent Technologies (Santa Clara, CA, USA) and Peptide Institute (Osaka, Japan), respectively. Recombinant human annexin A2 and human legumain were purchased from ProSpec-Tany TechnoGene (Ness Ziona, Israel) and R\&D Systems (Minneapolis, USA), respectively. Balbc mice were purchased from Sankyo Lab. (Tokyo, Japan). All other chemicals were of analytical grade and purchased from Wako Pure Chemicals (Osaka, Japan).

\subsection{Preparation of anti-annexin A2 antibodies}

Annexin A2 purified from the bovine kidney was used as an immunogen to establish rabbit anti-annexin A2 polyclonal antisera. Briefly, one hundred fifty $\mu$ g of purified annexin A2 dissolved in PBS was first injected into rabbits and then injected five times as booster injections at 10-day intervals. Antigen solutions were mixed with equal volumes of complete adjuvant and with incomplete adjuvant and were used for the initial and subsequent injections, 
respectively. An IgG fraction of an anti-annexin A2 antibody was purified from antisera using a protein A-Sepharose column.

\subsection{Western blotting and antibodies}

To examine the degradation levels of annexin A2 and expression levels of legumain in the mouse kidney, proteins were extracted from cultured cells or from the mouse kidney in a solution containing $50 \mathrm{mM}$ sodium citrate buffer $\mathrm{pH}$ 5.0. Proteins were then separated on a 12.5\% polyacrylamide gel and subjected to Western blotting with respective antibodies. Proteins on the membrane were reacted with an IRDye 800- (Rockland, Philadelphia, PA, USA) or Alexa Fluor 680-conjugated secondary antibody (Molecular Probes, Eugene, OR, USA) and visualized by using an infrared imaging system (Odyssey, LI-COR, Lincoln, NE, USA). Antibodies used were anti-legumain (1:500, Santa Cruz, CA, USA) and anti-annexin II (1:1000) antibodies.

\subsection{Inhibition of annexin A2 degradation in bovine kidney homogenates}

To examine annexin A2 degradation by legumain under an acidic condition, $8 \mu \mathrm{g}$ of bovine kidney homogenates was incubated in a solution containing $50 \mathrm{mM}$ sodium citrate (pH 5.0) and $2 \mathrm{mM}$ 2-mercaptoethanol in the presence or absence of $1 \mathrm{mM}$ N-ethylmaleimide for 30 min at $37^{\circ} \mathrm{C}$, separated on a $12.5 \%$ SDS-polyacrylamide gel, and subjected to Western blotting with an anti-annexin A2 antibody.

\subsection{Preparation of siRNAs}

Three siRNAs targeting mouse legumain (B-Bridge, Cat\#: SMF27A-1537-1, SMF27A-1537-2 and SMF27A-1537-3) were used. Nucleotide sequences of the siRNAs used were as follows: LGMN (SMF27A-1537-1; $\quad$ siRNA1): $\quad$ sense, 
5'-GGGCAAAGGGUCUGGAAAATT-3'

and

antisense,

5'-UUUUCCAGACCCUUUGCCCTT-3'; LGMN (SMF27A-1537-2; siRNA2): sense,

5'-GGGAAACUGCUGAGAGACATT-3'

and

antisense,

5'-UGUCUCUCAGCAGUUUCCCTT-3'; and LGMN (SMF27A-1537-3; siRNA3): sense, 5'GGGAAGGAAUCGUCUGAGATT-3' and antisense, 5'-

UCUCAGACGAUUCCUUCCCTT-3'. Nucleotide sequences of the negative control siRNA were as follows: sense, 5'-CGUACGCGGAAUACUUCGATT-3' and antisense, 5'-UCGAAGUAUUCCGCGUACGTT-3'.

\subsection{Formation of transfection complex}

siRNAs were transfected into NIH3T3 cells by using LipoTrust ${ }^{\mathrm{TM}} E X$ Oligo (Hokkaido System Science). A vial of LipoTrust was reconstituted of $1 \mathrm{ml}$ of nuclease-free water, and 10 $\mu \mathrm{l}$ of LipoTrust solution was mixed with 100 pmol of siRNA. After incubation of the mixture for 20 min at room temperature, legumain siRNA-lipoplex was formed. For in vivo study, a vial of LipoTrust was reconstituted of $1 \mathrm{ml}$ of nuclease-free water containing 1.6, 3.2 and 6.4 nmol siRNAs. After incubation of the mixture for $20 \mathrm{~min}$ at room temperature, legumain siRNA-lipoplex was formed.

\subsection{Characterization of legumain siRNA-lipoplex}

The size dispersion of legumain siRNA-lipoplex was determined by N4 Plus (Beckman Coulter, Miami, FL, USA) and the zeta potential was measured using a Zeta Potential Analyzer Ver.3.47 (Brookhaven Instruments Corp., NY, USA).

\subsection{Cell culture and knockdown of legumain expression in vitro}

NIH3T3 cells were cultured in Dulbecco's modified Eagle’s medium supplemented with 10\% 
calf serum at $37^{\circ} \mathrm{C}$ in a humidified atmosphere containing $5 \% \mathrm{CO}_{2}$. The cells were transfected with Legumain siRNA-lipoplex. Forty-eight hours after transfection, cells were collected and their total RNAs were extracted.

\subsection{Knockdown of mouse legumain expression in vivo}

C57BL/6 mice were purchased from Sankyo Lab. (Tokyo, Japan). Eighty $\mu$ l of each of the legumain siRNA-lipoplex solutions (1.6 and 3.2 and $6.4 \mathrm{nmol} / \mathrm{ml}$ siRNA) was injected into mouse tail veins. Lipoplex solution containing negative control siRNA was also injected. At 48 hours after injection, mouse kidneys were extracted and their total RNAs and proteins were extracted. All animal experiments were carried out in accordance with the National Institutes of Health Guide for the Care and Use of Laboratory Animals, and the protocols were approved by the Committee for Animal Research at Hokkaido University (permit number: 08-0484).

\subsection{Quantification of mRNA level by reverse transcription polymerase chain reaction}

Tissues were dissected and quickly frozen in an RNAlater solution. Total RNAs were prepared from approximately $20 \mathrm{mg}$ of the kidney using an RNeasy mini kit, and quality of RNA was examined using Bioanalyzer. Reverse transcription was carried out in a mixture containing 500 ng of total RNAs, Superscript III and specific primers under the conditions of $96^{\circ} \mathrm{C}$ for $15 \mathrm{~min}, 32$ cycles of $96^{\circ} \mathrm{C}$ for $30 \mathrm{sec}, 60^{\circ} \mathrm{C}$ for $30 \mathrm{sec}, 72^{\circ} \mathrm{C}$ for $30 \mathrm{sec}$ and $72^{\circ} \mathrm{C}$ for 5 min by using a QuantiTect SYBER Green kit (Qiagen) and Opticon II real-time PCR analyzer (Bio-Rad, Hercules, CA, USA). $\beta$-actin (ACTB) mRNA was also amplified and used as an internal control.

\subsection{Proteolytic activity of legumain}


Enzyme activity of legumain was examined by measuring the fluorometrical number (excitation, $380 \mathrm{~nm}$; emission, $440 \mathrm{~nm}$ ) of liberation of 7-amino-4-methylcoumarin (AMC) in a mixture containing $10 \mu \mathrm{l}$ of $10 \mathrm{mM}$ Z-Ala-Ala-Asn-MCA, $100 \mu \mathrm{l}$ of $0.5 \mathrm{M}$ sodium citrate buffer $\mathrm{pH}$ 5.0, $5 \mu \mathrm{l}$ of $1 \mathrm{M}$ 2-mercaptoethanol, $20 \mu \mathrm{l}$ of enzyme solution and water $(18 \mathrm{~m} \Omega)$ in a total volume of $1 \mathrm{ml}$. After incubation of the mixture at $37^{\circ} \mathrm{C}$ for $30 \mathrm{~min}, 2 \mathrm{ml}$ of $0.2 \mathrm{M}$ acetic acid was added to the mixture to stop the reaction. One unit of activity was defined as the amount of enzyme that hydrolyzed $1 \mu \mathrm{mol}$ of the substrate per min.

\subsection{Statistical analyses}

Data are expressed as means \pm S.D for mouse experiments. Statistical analyses were performed using analysis of variance (one-way ANOVA) followed by unpaired Student's t-test. For comparison of multiple samples, the Tukey-Kramer test was used.

\section{Results}

\subsection{Effect of legumain siRNAs on legumain expression in NIH3T3 cells}

To examine the effect of legumain siRNAs on the expression of legumain mRNA, three siRNA-lipoplexes targeting legumain were transfected into NIH3T3 cells and the expression of legumain mRNA was examined by real-time PCR. As shown in Fig. 1A, the expression level of legumain mRNA was decreased by legumain siRNA-lipoplexes compared to that in control siRNA-transfected cells. The degrees of knockdown of legumain mRNA by legumain siRNA1, 2 and 3-lipoplexes were 42, 55 and 66\%, respectively. The siRNA \#3 having the strongest knockdown activity toward legumain expression was therefore used for further studies.

\subsection{Effect of legumain siRNAs on legumain expression and activity in the mouse kidney}


To examine the effect of legumain siRNAs on legumain expression in vivo, legumain siRNA3-lipoplex and negative siRNA-lipoplex were injected into the mouse tail veins and the expression level of legumain in the mouse kidney was examined by real-time PCR. As shown in Fig. 1B, legumain expression level was decreased by legumain siRNA-lipoplex containing $3.2 \mathrm{nmol} / \mathrm{ml}$ siRNA3 to $80 \%$ of that by negative siRNA-lipoplex, but legumain siRNA-lipoplex containing $6.4 \mathrm{nmol} / \mathrm{ml}$ siRNA3 did not show a knockdown effect. To determine the factor(s) that affects knockdown efficiency of legumain siRNA-lipoplexes, zeta potential and size dispersion of legumain siRNA-lipoplexes containing 1.6, 3.2 and $6.4 \mathrm{nmol}$ of siRNA3 were measured using a Zeta Potential Analyzer and N4 Plus, respectively. The results showed that the size and zeta potential of legumain siRNA-lipoplex containing 6.4 $\mathrm{nmol} / \mathrm{ml}$ of siRNA3 were increased more than 2-fold and decreased by $20 \%$, respectively, compared with those of legumain siRNA-lipoplex complex containing $3.2 \mathrm{nmol} / \mathrm{ml}$ of siRNA3 (Figs. 1C and 1D).

The effect of legumain siRNA3-lipoplex on legumain activity in mouse tissues was then examined. As shown in Fig. 1E, legumain activity in the kidney, but not that in the liver or spleen, was decreased by injection of legumain siRNA-lipoplex containing $3.2 \mathrm{nmol} / \mathrm{ml}$ of siRNA3 to about $40 \%$ of that in negative siRNA-lipoplex-injected mice.

\subsection{Degradation of annexin A2 by legumain in the mouse kidney}

To examine degradation of annexin A2 by cysteine proteases under an acidic condition, bovine kidney homogenates were incubated in a mixture containing $1 \mathrm{mM}$ N-ethylmaleimide (NEM), an inhibitor of cysteine proteases, and proteins in the mixture were separated on a polyacrylamide gel and subjected to Western blotting with an anti-annexin A2 antibody. Purified annexin A2, which lacks the N-terminal region, was also loaded on the gel as a positive control. As shown in Fig. 2A, annexin A2 degradation was observed in the bovine 
kidney homogenate but was inhibited by the presence of NEM, indicating that annexin A2 degradation was carried out by a cysteine protease(s). Since legumain is a cysteine protease, the possibility that legumain degrades annexin A2 in vivo was examined. To do that, legumain siRNA3-lipoplex and negative siRNA-lipoplex were injected into mouse tail veins. Forty-eight hrs after injection, proteins in the kidney were analyzed by Western blotting with anti-legumain, anti-annexin A2 and anti-actin antibodies. Two bands of annexin A2 corresponding to full-sized and cleaved annexin A2 were obtained (Fig. 2B), and the levels of cleaved and full-sized annexin A2 in legumain siRNA3-lipoplex-injected mice were decreased and increased to about $60 \%$ and $30 \%$ of those in negative siRNA-lipoplex-injected mice, respectively (Figs. 2C and 2D), suggesting that legumain directly cleaves annexin A2 or affects cleavage of annexin A2.

\subsection{Direct cleavage of annexin A2 by legumain in vitro}

To examine degradation of annexin A2 by legumain, $6 \mathrm{nmol}$ of recombinant human annexin A2 was reacted with $0.12,0.24$ and $1.2 \mathrm{nmol}$ of recombinant human legumain and subjected to Western blotting. As shown in Figs. 3A and 3B, annexin A2 was cleaved by recombinant legumain in a dose-dependent manner, indicating that legumain directly cleaved annexin A2.

\section{Discussion}

In this study, we showed that legumain siRNA-lipoplex containing $3.2 \mathrm{nmol} / \mathrm{ml}$ siRNA3 knocked down legumain mRNA expression by about 60\% and 20\% in NIH3T3 cells and in the mouse kidney, respectively (Figs. 1A and 1B). These results suggest that legumain siRNA was delivered to the kidney and that more than $40 \%$ of legumain siRNA3-lipoplex was degraded during a period of 48 hours after injection into mouse tail veins. Legumain siRNA-lipoplex containing $6.4 \mathrm{nmol} / \mathrm{ml}$ siRNA3, on the other hand, did not affect the 
expression level of legumain (Fig. 1B). The size and zeta potential of legumain siRNA-lipoplex containing $6.4 \mathrm{nmol} / \mathrm{ml}$ of siRNA3 were increased more than 2-fold and decreased by $20 \%$ compared to those of legumain siRNA-lipoplex complex containing 3.2 nmol/ml of siRNA3 (Fig. 1C, D). These results indicate that the size and zeta potential of legumain siRNA3-lipoplex are important factors to obtain an efficient knockdown effect in the mouse kidney. We also found that legumain activity toward the synthetic substrate Z-Ala-Ala-Asn-MCA in the mouse kidney was decreased by about $40 \%$ of that in negative siRNA-lipoplex-injected mice, while legumain activities were not changed in the liver and spleen (Fig. 1E), suggesting that the knockdown effect of legumain siRNA3-lipoplex is tissue-specific.

Futhermore, we found that the cleavage level of annexin A2 was decreased in the legumain siRNA-treated mouse kidney (Fig. 2) and that legumain directly degrades annexin A2 (Fig. 3). These results suggest that legumain siRNA inhibited annexin A2 degradation under an acidic condition through the reduced expression of legmain in vivo. It has been reported that the $\mathrm{N}$-terminal region of annexin A2 was cleaved by plasmin under the condition of neutral $\mathrm{pH}$ [21]. We previously suggested that legumain cleaves annexin A2 under an acidic $\mathrm{pH}$ condition, which resembles conditions in the endosome or lysosome [3]. Annexin A2 was identified as one of the AQP2-associated proteins [15]. The $\mathrm{N}$-terminal region of annexin A2 is required for cAMP-induced AQP2 exocytosis in renal cells [16], and phosphorylation of the N-terminal region is essential for proper endosomal association [18]. It is therefore thought that legumain regulates functions of annexin A2, including AQP2 trafficking and endosomal association-mediated cleavage of annexin A2 in the endosome.

In conclusion, we found that legumain siRNA3-lipoplex decreased the expression of legumain mRNA and protein and its proteolytic activity in vivo, thereby inhibiting annexin A2 degradation by legmain under an acidic condition. Although legumain was suggested to 
regulate the function of annexin A2 in the endosome and lysosome in the kidney, future studies are needed to reveal the molecular mechanism underlying the regulation by legumain of annexin A2 functions such as fusion and trafficking of membrane proteins.

Abbreviations: Z, benzyloxycarbonyl; MCA, methylcumarinamide; siRNA-lipoplex, siRNA-cationic liposome complex; NEM, N-ethyl maleimide; 2-ME, 2-mercaptoetanol; PBS, phosphate buffered saline.

\section{Acknowledgements}

We thank Yayoi Shimizu for her technical assistance. This work was supported by the Scientific Research Fund from Hokkaido System Science and Hokkaido Wako Junyaku.

\section{References}

[1] S. Ishii, Legumain: asparaginyl endopeptidase, Methods Enzymol. 244 (1994) 604-615.

[2] J.M. Chen, P.M. Dando, R.A.E. Stevens, M. Fortunato, A.J. Barrett, Cloning and expression of mouse legumain, a lysosomal endopeptidase, Biochem. J. 335 (1998) 111-117.

[3] T. Yamane, K. Takeuchi, Y. Yamamoto, Y.H. Li, M. Fujiwara, K. Nishi, S. Takahashi, I., Ohkubo, Legumain from bovine kidney: its purification, molecular cloning, immunohistochemical localization and degradation of annexin II and vitamin D-binding protein, Biochim. Biophys. Acta 1596 (2002) 108-120.

[4] Y. Morita, H. Araki, T. Sugimoto, K. Takeuchi, T. Yamane, T. Maeda, Y. Yamamoto, K.Nishi, M. Asano, K. Shirahama-Noda, M. Nishimura, T. Uzu, I. Hara-Nishimura, D. Koya, A. Kashiwagi, I. Ohkubo, Legumain/asparaginyl endopeptidase controls extracellular matrix remodeling through the degradation of fibronectin in mouse renal 
proximal tubular cells, FEBS Lett. 581 (2007) 1417-1424.

[5] S.J. Oosterling, G.J. van der Bij, G.A. Meijer, C.W. Tuk, E. van Garderen, N. van Rooijen, S. Meijer, J.R. van der Sijp, R.H. Beelen, M. van Egmond, Macrophages direct tumour histology and clinical outcome in a colon cancer model, J. Pathol. 207 (2005) 147-155.

[6] C. Liu, C. Sun, H. Huang, K. Janda, T. Edgington, Overexpression of legumain in tumors is significant for invasion/metastasis and a candidate enzymatic target for prodrug therapy, Cancer Res. 63 (2003) 2957-2964.

[7] R.V. Murthy, G. Arbman, J. Gao, G.D. Roodman, X.F. Sun, Legumain expression in relation to clinicopathologic and biological variables in colorectal cancer, Clin. Cancer Res. 11 (2005) 2293-2299.

[8] S.B. Moparthi, G. Arbman, A. Wallin, H. Kayed, J. Kleeff, H. Zentgraf, X.F. Sun, Expression of MAC30 protein is related to survival and biological variables in primary and metastatic colorectal cancers, Int. J. Oncol. 30 (2007) 91-95.

[9] Y. Luo, H. Zhou, J. Krueger, C. Kaplan, S.H. Lee, C. Dolman, D. Markowitz, W. Wu, C. Liu, R.A. Reisfeld, R. Xiang, Targeting tumor-associated macrophages as a novel strategy against breast cancer, J. Clin. Invest. 116 (2006) 2132-2141.

[10] M. Papaspyridonos, A. Smith, K.G. Burnand, P. Taylor, S. Padayachee, K.E. Suckling, C.H. James, D.R. Greaves, L. Patel, Novel candidate genes in unstable areas of human atherosclerotic plaques, Arterioscler Thromb. Vasc. Biol. 26 (2006) 1837-1844.

[11] C.B. Chan, M. Abe, N. Hashimoto, C. Hao, I. R. Williams, X. Liu, S. Nakao, A. Yamamoto, S.Y. Li, I. Hara-Nishimura, M. Asano, K. Ye, Mice lacking asparaginyl endopeptidase develop disorders resembling hemophagocytic syndrome, Proc. Nat. Acad. Sci. USA. 106 (2009) 468-473.

[12] K.L. Mattock, P.J. Gough, J. Humphries, K. Burnand, L. Patel, K. E. Suckling, F. Cuello, C. Watts, M. Gautel, M. Avkiran, A. Smith, Legumain and cathepsin-L expression in human 
unstable carotid plaque, Atherosclerosis 208 (2010) 83-89.

[13] D.M. Waisman, Annexin II tetramer: structure and function, Mol. Cell. Biochem. 149/150 (1995) 301-322.

[14] A. Pol, D. Ortega, C. Enrich, Identifcation of cytoskeletonassociated proteins in isolated rat liver endosomes, Biochem J. 327 (1997) 741-746.

[15] Y. Noda, S. Horikawa, Y. Katayama, S. Sasaki, Identification of a multiprotein "motor" complex binding to water channel aquaporin-2, Biochem. Biophys. Res. Commun. 330 (2005) 1041-1047.

[16] G. Tamma, G. Procino, M.G. Mola, M. Svelto, G. Valenti, Functional involvement of Annexin-2 in cAMP induced AQP2 trafficking, Pflugers Arch. 456 (2008) 729-736.

[17] M. Zibouche, M. Vincent, F. Illien, J. Gallay, J.A. Sanmartin, The N-terminal domain of annexin 2 serves as a secondary binding site during membrane bridging, J. Biol. Chem. 283 (2008) 22121-22127.

[18] E. Morel, J. Gruenberg, Annexin A2 binding to endosomes and functions in endosomal transport are regulated by tyrosine 23 phosphorylation, J. Biol. Chem. 284 (2009) 1604-1611.

[19] K.A. Whitehead, R Langer, D.G. Anderson, Knocking down barriers: advances in siRNA delivery. Nat. Rev. Drug Discov. 8 (2009) 129-138.

[20] T Kato, A Natsume, H Toda, H Iwamizu1, T Sugita, R Hachisu, R Watanabe, K Yuki, K Motomura1, K Bankiewicz, T Wakabayashi, Efficient delivery of liposome-mediated MGMT-siRNA reinforces the cytotoxity of temozolomide in GBM-initiating cells. Gene Therapy 17 (2010) 1363-1371.

[21] P.A. Madureira, A.P. Surette, K.D. Phipps, M.A. Taboski, V.A. Miller, D.M. Waisman, The role of the annexin A2 heterotetramer in vascular fibrinolysis, Blood 118 (2011) 4789-4797. 


\section{Figure legends}

Fig. 1. Effect of legumain siRNAs on legumain expression and activity

A. Lipoplexes containing three kinds of legumain siRNAs or negative control siRNA were transfected into NIH3T3 cells. At 48 hours after transfection, the expression levels of legumain and $\beta$-actin mRNA were examined by real-time PCR. Proteins extracted from NIH3T3 cells were also analyzed by Western blotting with anti-legumain and $\beta$-actin antibodies. Statistical analyses were carried out using the Tukey-Kramer test. Significance: ${ }^{*} p<0.05,{ }^{* *} p<0.01$ versus negative control siRNA.

B. Legumain siRNA-lipoplex solutions containing 1.6, 3.2 and $6.4 \mathrm{nmol} / \mathrm{ml}$ siRNA or 1.6 $\mathrm{nmol} / \mathrm{ml}$ control siRNA were injected into mouse tail veins. At 48 hours after injection, the mouse kidneys were extracted and their expression levels of mRNA and protein of legumain were examined by real-time PCR and by Western blotting.

C and D. Size dispersion of legumain siRNA-lipoplex was determined by N4 Plus, and the zeta potential was measured using a Zeta Potential Analyzer Ver.3.47.

E. Legumain siRNA-lipoplex solutions containing $3.2 \mathrm{nmol} / \mathrm{ml}$ siRNA or $1.6 \mathrm{nmol} / \mathrm{ml}$ negative siRNA-lipoplex were injected to mouse tail veins. At 48 hours after injection, the mouse liver, spleen and kidney were extracted and their proteins were extracted. Their legumain activities were then measured by fluorometrical assays against Z-Ala-Ala-Asn-MCA. Statistical analyses were carried out using the Tukey-Kramer test. ${ }^{* *} p<0.01, * p<0.05$, N.S., not significant

Fig. 2. Effect of knockdown of mouse legumain expression on annexin A2 degradation in vivo.

A. Eight $\mu$ g of bovine kidney homogenate was incubated with or without $1 \mathrm{mM}$ NEM in a solution containing $50 \mathrm{mM}$ sodium citrate buffer (pH 5.0) and $2 \mathrm{mM}$ 2-ME. Annexin A2 was 
analyzed by Western blotting with an anti-legumain antibody. Lane C indicates a band corresponding to purified bovine kidney annexin A2 lacking the N-terminal region.

B. Lipoplex solutions containing $3.2 \mathrm{nmol} / \mathrm{ml}$ legumain and negative control siRNAs were injected into mouse tail veins. At 48 hours after injection, the mouse kidneys were extracted and the expression levels of legumain, annexin A2 and $\beta$-actin were examined by Western blotting. Lane C indicates a band corresponding to purified bovine kidney annexin A2 lacking the $\mathrm{N}$-terminal region.

$\mathrm{C}$ and D. Intensity of cleaved and non-cleaved annexin A2 bands shown in Fig. 2B was quantified. Statistical analyses were carried out using Student’s $t$-test. Significance: ${ }^{*} p<0.05$, ${ }^{* *} p<0.01$

Fig. 3. Direct cleavage of annexin A2 by legumain

A. Six nmol of recombinant human annexin A2 was reacted with $0.12,0.24$ and $1.2 \mathrm{nmol}$ of recombinant human legumain in a solution containing $50 \mathrm{mM}$ sodium citrate buffer (pH 5.0) and $2 \mathrm{mM}$ 2-ME at $37^{\circ} \mathrm{C}$ for $30 \mathrm{~min}$. Annexin A2 was analyzed by Western blotting with an anti-annexin A2 antibody.

B. Intensity of the cleaved annexin A2 band shown in Fig. 3A was quantified. Statistical analyses were carried out using the Tukey-Kramer test. Significance: ${ }^{*} p<0.05$ 


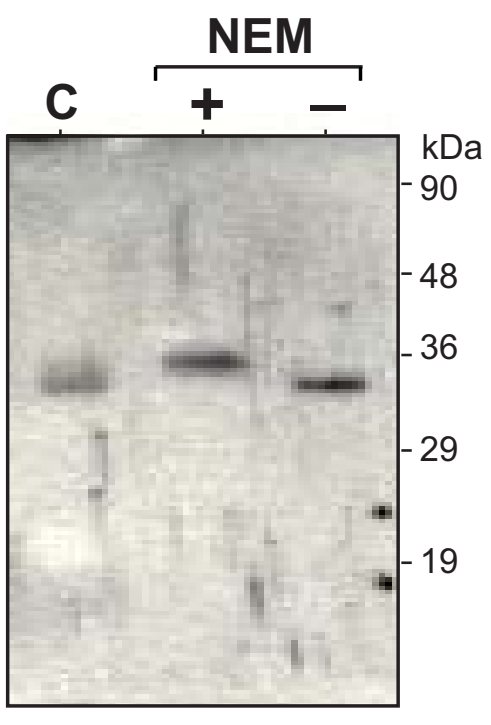

\section{B}

Legumain siRNA $\quad-\quad+$

Legumain

C

Annexin A2

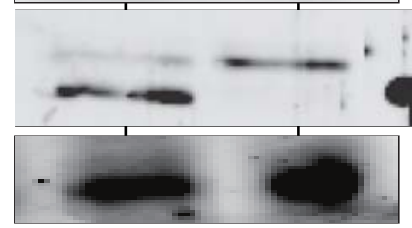

Actin

C

D
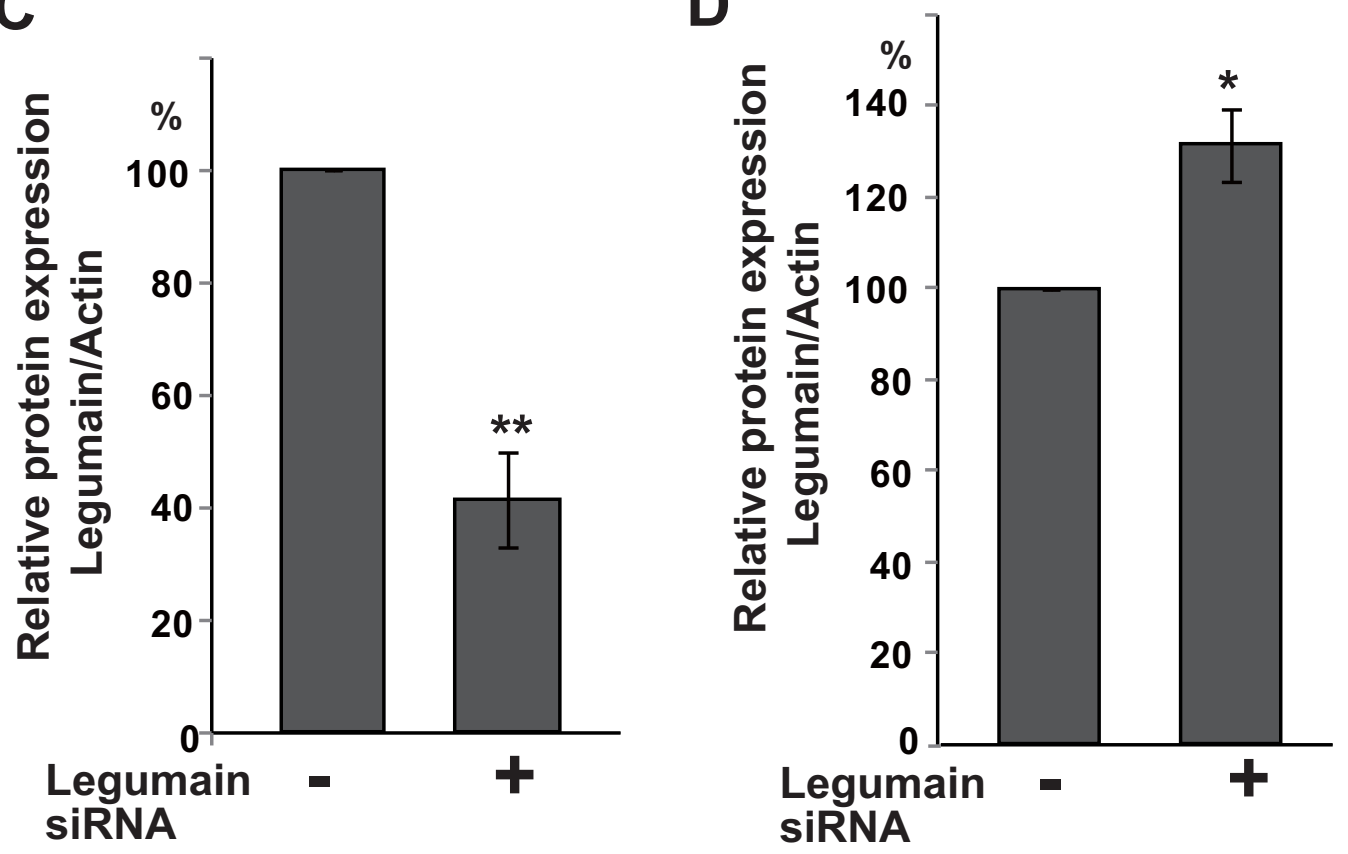

Fig. 2 
A
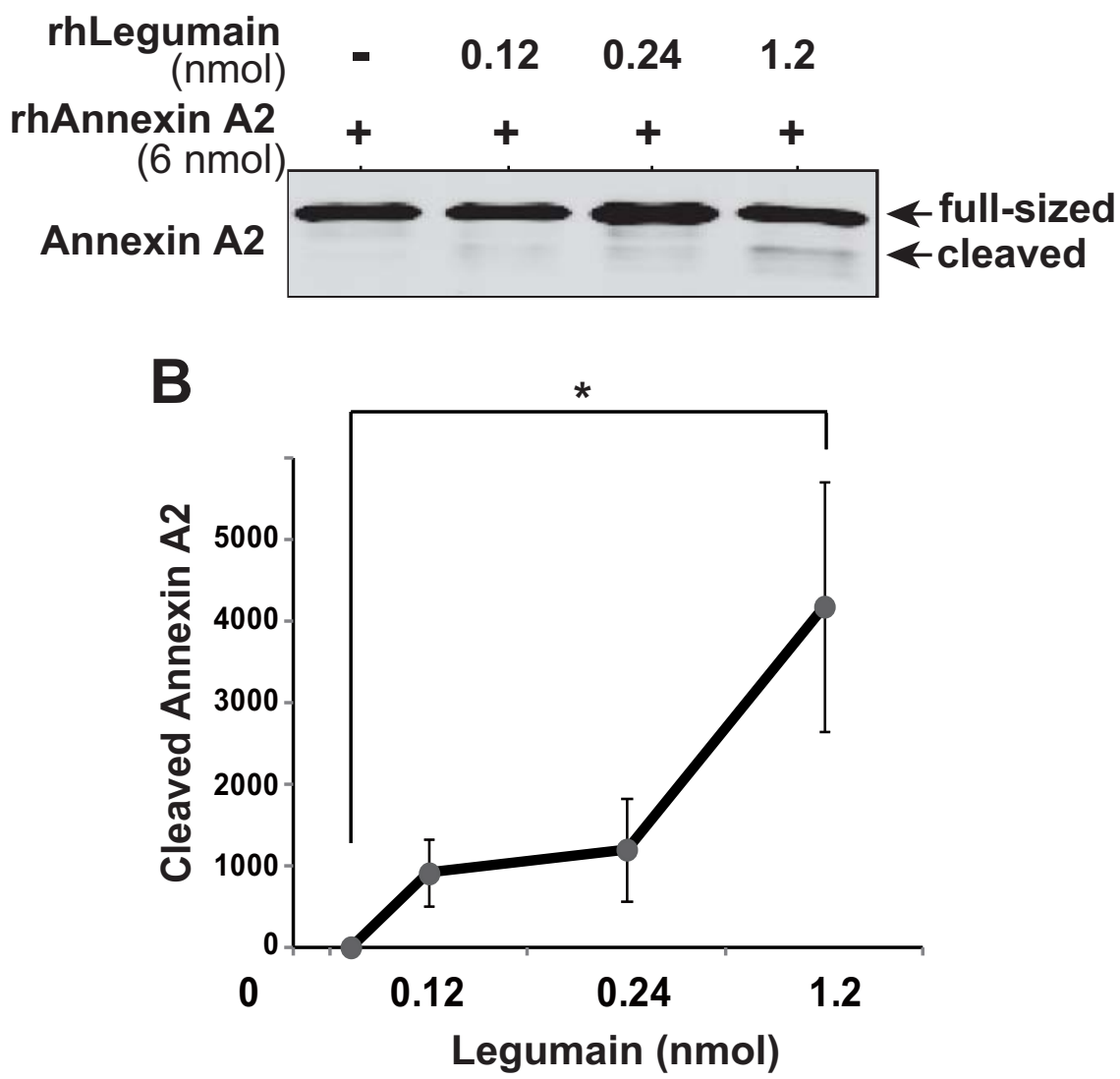